\title{
Expression of flTF and asTF splice variants in various cell strains and tissues
}

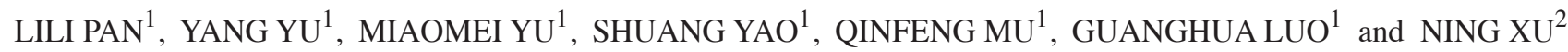 \\ ${ }^{1}$ Comprehensive Laboratory, Changzhou Key Lab of Individualized Diagnosis and Treatment Associated with High Technology \\ Research, The Third Affiliated Hospital of Soochow University, Changzhou, Jiangsu 213003, P.R. China; ${ }^{2}$ Division of Clinical \\ Chemistry and Pharmacology, Department of Laboratory Medicine, Lund University Hospital, S-221 85 Lund, Sweden
}

Received June 15, 2018; Accepted November 28, 2018

DOI: $10.3892 / \mathrm{mmr} .2019 .9843$

\begin{abstract}
Tissue factor (TF) expressed at the protein level includes two isoforms: The membrane-bound full-length TF (fITF) and the soluble alternatively spliced TF (asTF). flTF is the major thrombogenic form of TF, whereas asTF is more closely associated with tumor growth, angiogenesis, metastasis and cell growth. In order to further investigate the different expression and functions of TF splice variants, the expression of these two splice variants were detected in numerous cell strains and tissues in the present study. Quantitative polymerase chain reaction was used to measure the transcript levels of the TF variants in 11 human cell lines, including cervical cancer, breast cancer, hepatoblastoma, colorectal cancer and umbilical vein cells, and five types of tissue specimen, including placenta, esophageal cancer, breast cancer, cervical cancer (alongside normal cervical tissues) and non-small cell lung cancer (alongside adjacent and normal tissues). Furthermore, the effects of chenodeoxycholic acid (CDCA) and apolipoprotein $\mathrm{M}$ (apoM) on the two variants were investigated. The results demonstrated that flTF was the major form of TF, and the mRNA expression levels of flTF were higher than those of asTF in all specimens tested. CDCA significantly upregulated the mRNA expression levels of the two variants. Furthermore, overexpression of apoM promoted the expression levels of asTF in Caco-2 cells. The mRNA expression levels of asTF in cervical cancer tissues were significantly higher than in the
\end{abstract}

Correspondence to: Dr Guanghua Luo, Comprehensive Laboratory, Changzhou Key Lab of Individualized Diagnosis and Treatment Associated with High Technology Research, The Third Affiliated Hospital of Soochow University, 185 Juqian Street, Changzhou, Jiangsu 213003, P.R. China

E-mail: shineroar@163.com

Dr Ning Xu, Division of Clinical Chemistry and Pharmacology, Department of Laboratory Medicine, Lund University Hospital, Klinikgatan 17, S-221 85 Lund, Sweden

E-mail: ning.xu@med.lu.se

Key words: tissue factor, variants, tumor, quantitative polymerase chain reaction corresponding normal tissues. To the best of our knowledge, the present study is the first to compare the expression of fITF and asTF in various samples. The results demonstrated that CDCA and apoM may modulate TF isoforms in different cell lines, and suggested that asTF may serve a role in the pathophysiological mechanism underlying cervical cancer development. In conclusion, the TF isoforms serve important and distinct roles in pathophysiological processes.

\section{Introduction}

Tissue factor (TF) is a 47-kD transmembrane cell-surface glycoprotein, which is primarily known as the initiator of the blood coagulation cascade (1). Human TF is genetically encoded by the TF gene, which is transcribed to TF premature mRNA. Alternative splicing of TF results in three naturally occurring protein isoforms: Full-length (fl)TF, alternatively spliced (as)TF and TF-A. asTF and fITF serve important and distinct roles in various biological processes that involve vessel formation and maturation, and initiation of the blood coagulation cascade (2). asTF, which arises from exclusion of the fifth exon of the primary TF transcript, exhibits low prothrombogenic potential but is more closely associated with tumor growth, angiogenesis, metastasis and cell growth (3-5). TF-A, another splice variant, is only expressed at the mRNA level in a number of cancer cell lines and in endothelial cells (Fig. 1). To the best of our knowledge, the biological function of TF-A mRNA is currently unknown; therefore, this splice variant was not taken into account in the present study (6-8).

Few studies regarding TF expression have discriminated between flTF and asTF; therefore, it is necessary to investigate the expression status of these two isoforms in different diseases. Bile acids are strong signaling molecules that are capable of influencing various biological processes, including inflammation, apoptosis, cancer progression and atherosclerosis. Chenodeoxycholic acid (CDCA) is a bile acid that has been demonstrated to enhance ectopic vessel formation (9). Similarly, apolipoprotein M (apoM), which was discovered by Xu and Dahlbäck in 1999 (10), is mainly located in high-density lipoprotein in the blood and has been demonstrated to be associated with tumor growth, atherosclerosis and thrombosis (11-13). In order to investigate the association between $\mathrm{TF}$ variants and $\mathrm{CDCA} / \mathrm{apoM}$, the difference in the 
expression of TF variants in various cell strains and tissues was examined in the present study. The results of this study may contribute to further studies on the function and mechanism of TF in associated diseases.

\section{Materials and methods}

Cell lines and cell culture. Human cervical cancer cell lines [C-33A, human papilloma virus (HPV)-negative; HeLa, HPV18-positive; and SiHa, HPV16-positive], human breast cancer cell lines (ZR-75-1, luminal A subtype; MCF-7, luminal A subtype; BT-474, luminal B subtype; MDA-MB-468, basal-like subtype; and MDA-MB-231, basal-like subtype), a human hepatoblastoma cell line (HepG2), a human colorectal cancer cell line (Caco-2) and a human umbilical vein cell line (EA.hy926) were purchased from the Cell Bank of Type Culture Collection of the Chinese Academy of Sciences (Shanghai, China). All cells were cultured according to their respective conditions and maintained in the Comprehensive Laboratory of The Third Affiliated Hospital of Soochow University (Changzhou, China).

The C-33A, HeLa, SiHa, Caco-2 and HepG2 cells were cultured in minimum essential medium (Gibco; Thermo Fisher Scientific, Inc., Waltham, MA, USA), and the MCF-7 and EA.hy926 cells were maintained and cultured in Dulbecco's modified Eagle's medium (Gibco; Thermo Fisher Scientific, Inc.). The BT-474 and ZR-75-1 cells were cultured in RPMI-1640 media (Gibco; Thermo Fisher Scientific, Inc.), whereas the MDA-MB-468 and MDA-MB-231 cells were cultured in Leibovitz's L-15 medium (Gibco; Thermo Fisher Scientific, Inc.). All of the complete media were supplemented with 10\% fetal bovine serum (Gibco; Thermo Fisher Scientific, Inc.) and $1 \%$ penicillin/streptomycin, and $10 \%$ non-essential amino acid solution was added for the culture of cervical cancer cell lines. All cells were incubated at $37^{\circ} \mathrm{C}$ in an atmosphere containing $5 \% \mathrm{CO}_{2}$, with the exception of the MDA-MB-468 and MDA-MB-231 cells, which were cultured at $37^{\circ} \mathrm{C}$ in a humidified atmosphere containing $100 \%$ air.

The HepG2 and EA.hy926 cells were seeded in 6-well plates with the concentration of $2 \times 10^{5}$ cells/well, and allowed to grow to $80-90 \%$ confluence. Subsequently, they were washed and incubated in serum-free medium containing $50 \mu \mathrm{M}$ CDCA (Sigma-Aldrich; Merck KGaA, Darmstadt, Germany) with $0.1 \%$ ethanol for $24 \mathrm{~h}$ at $37^{\circ} \mathrm{C}$. Total RNA was then extracted.

The apoM coding sequence was obtained by polymerase chain reaction (PCR) amplification from human genomic DNA (forward primer, 5'-GAGGATCCCCGGGTACCGGTCGCC ACCATGTTCCACCAA ATT TGGGCAGC-3'; reverse primer, 5'-TCCTTGTAGTCCATACCGTTATTGGACAGC TCACAGGCCTC-3') and was inserted into the Ubi-MCS3FLAG-CMVEGFP vector (cat. no. GV365; Shanghai Genechem Co., Ltd., Shanghai, China). Empty lentiviral vectors with green fluorescent protein (GFP) and lentivirus-mediated human apoM overexpression vectors with GFP were prepared by Shanghai GeneChem Co., Ltd. In brief, $20 \mu \mathrm{g} / \mathrm{ml}$ of GV365 vector, $15 \mu \mathrm{g} / \mathrm{ml}$ of pHelper 1.0 and $10 \mu \mathrm{g} / \mathrm{ml}$ of pHelper 2.0 (Shanghai Genechem Co., Ltd.) were cotransfected into 293T cells (Cell Bank of Type Culture Collection of the Chinese Academy of Sciences, Shanghai, China) with enhanced infection solution and polybrene (Shanghai Genechem Co., Ltd.,), cultured at $37^{\circ} \mathrm{C}$ for $48 \mathrm{~h}$ at $70 \%$ confluence. Lentiviral particles $\left[1 \times 10^{9}\right.$ transducing units $\left.(\mathrm{TU}) / \mathrm{ml}\right]$ were obtained from supernatants following centrifugation at a speed of $75,000 \mathrm{x} \mathrm{g}$ for $2 \mathrm{~h}$ at $4^{\circ} \mathrm{C}$. Subsequently, Caco-2 and EA.hy 926 cells at $50 \%$ confluence were incubated with the lentiviral vector (multiplicity of infection, $50 ; 3.75 \times 10^{8} \mathrm{TU}$ ) in dishes. After $12 \mathrm{~h}$, culture medium containing the lentivirus was aspirated from the wells and fresh complete medium was added. The expression intensity of GFP was observed 3 days later and apoM overexpression was confirmed using reverse transcriptionquantitative PCR (RT-qPCR). Caco-2 and EA.hy926 cells transfected with empty vectors (multiplicity of infection=50) were set up as the corresponding negative control (NC) groups.

Tissue sample collection. Placenta tissue specimens from 20 women (age, 24-33 years); esophageal cancer tissue specimens from 18 men and two women (age, 56-72 years); 14 breast cancer tissue specimens (age, 46-74); 34 cervical cancer specimens (age, 35-75 years) and 16 normal cervical control samples (age, 38-65 years); and non-small cell lung cancer (NSCLC) tissues and adjacent/normal tissues from seven men and nine women (age, 48-76 years) were collected at the Third Affiliated Hospital of Soochow University between July 2014 and September 2016. The patients had not received preoperative radiotherapy and/or chemotherapy. The experimental protocols were approved by the Institutional Ethics Committee of the Third Affiliated Hospital of Soochow University and all patients provided written informed consent for this study. All patients had undergone modified radical operations. All tissue samples including placental tissues, esophageal cancer tissues, breast cancer tissues, cervical cancer tissues, normal cervical tissues, NSCLC tissues and their adjacent/normal tissues were excised and quickly frozen in liquid nitrogen until subsequent analysis.

$R N A$ isolation and RT-qPCR. Total RNA was extracted from cells and tissues using the total RNA purification kit (Shenergy Biocolor Biological Science \& Technology Company, Shanghai, China), according to the manufacturer's protocol. cDNA was synthesized, according to the manufacturer's protocol, using the RevertAid First Strand cDNA Synthesis kit (Thermo Fisher Scientific, Inc.). Primers and TaqMan probes (labeled with carboxyfluorescein) for human fITF and asTF were designed using Primer Premier version 5.0 (Premier Biosoft International, Palo Alto, CA, USA) and were synthesized by Sangon Biotech Co., Ltd. (Shanghai, China) (Table I). The mRNA expression levels of flTF and asTF were quantified relative to the mRNA expression levels of GAPDH, and quantification was performed using a LightCycler ${ }^{\circledR} 480$ Instrument II (Roche Applied Science, Penzberg, Germany) in a final volume of $25 \mu \mathrm{l}$. PCR reactions, purchased from the Shenergy Biocolor BioScience and Technology Company (Shanghai, China), were performed using the following mixture: $2.5 \mu \mathrm{l} \mathrm{MgCl} 2(25 \mathrm{mM}$; with $4 \mu \mathrm{l} \mathrm{MgCl} 2$ in the asTF PCR reactions), $2.5 \mu 1$ PCR buffer (10X), $0.5 \mu 14 \mathrm{X}$ dNTP $(10 \mathrm{mM}), 0.25 \mu \mathrm{l}$ Taq DNA polymerase (5 units), $0.04 \mu \mathrm{l}$ each primer and probe $(100 \mu \mathrm{M}), 2 \mu \mathrm{l} \mathrm{cDNA}$ template (replaced by water in no template controls) and nuclease-free water to a final volume of $25 \mu \mathrm{l}$. Thermal cycling was performed under 
Table I. Primers and probes for reverse transcription-quantitative polymerase chain reaction.

\begin{tabular}{llll}
\hline Gene & \multicolumn{1}{c}{ Forward primer $\left(5^{\prime}-3^{\prime}\right)$} & \multicolumn{1}{c}{ Reverse primer (5'-3') } & \multicolumn{1}{c}{ Probe (5'FAM, 3'BHQ-1) } \\
\hline \multirow{2}{*}{ flTF } & TGATGTGGATAAAGGAGAA & CTACCGGGCTGTCTGTA & TTCAAGCAGTGATTCCCTCCC \\
& AACTACTGT & CTCTTC & GAACA \\
\multirow{2}{*}{ asTF } & ATCTTCAAGTTCAGGAAAGA & GCTCTGCCCCACTCCT & TTGGAGCTGTGGTATTTGTGG \\
& AATATTCTAC & GCC & TCATCATC \\
GAPDH & CAGGGCTGCTTTTACTC & CATGGGTGGAATCATATT & TGGATATTGTTGCCATCAATGA \\
& TGGT & GGAAC & CCCCT
\end{tabular}

asTF, alternatively spliced TF; flTF, full-length TF; TF, tissue factor.

TF gene \begin{tabular}{|l|l|l|l|l|l|l|l|l|l|l|l|}
\hline & 1 & & 2 & & 3 & & 4 & & 5 & & 6 \\
\hline
\end{tabular}

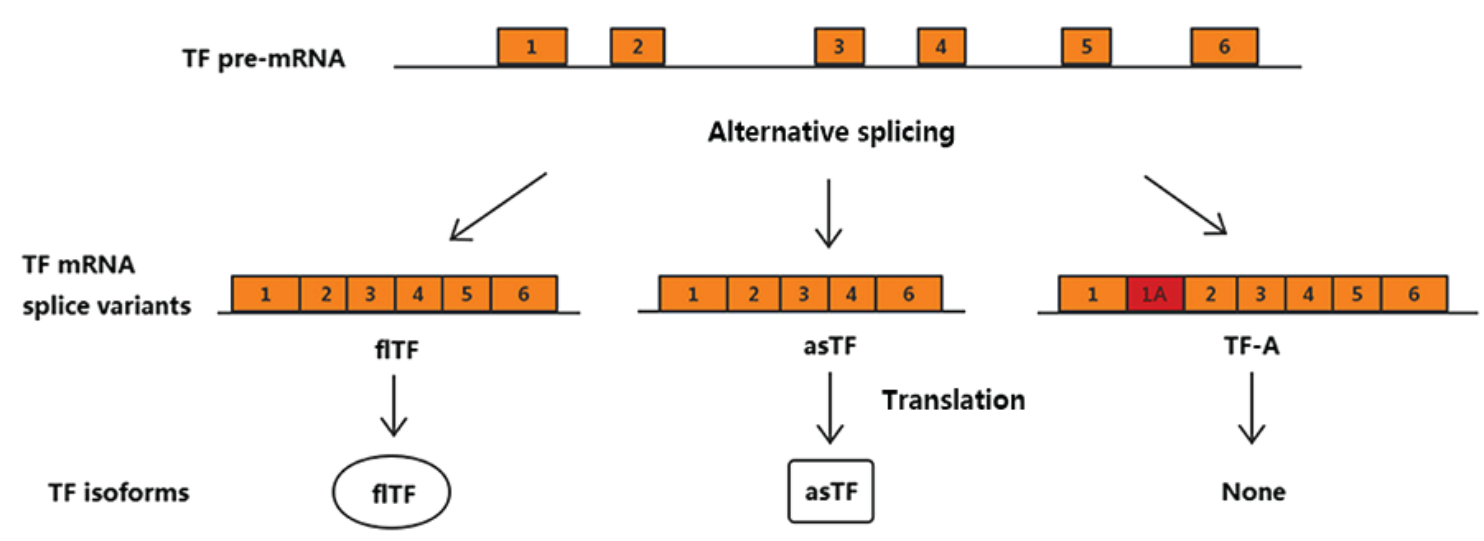

Figure 1. Schematic diagram of TF isoform expression. flTF consists of an intracellular domain, a transmembrane domain and an extracellular domain. asTF lacks the transmembrane domain and is therefore soluble. Retention of a part of intron 1 as alternative exon $1 \mathrm{~A}$ in the mature transcript leads to the generation of the third variant, TF-A. asTF, alternatively spliced TF; flTF, full-length TF; TF, tissue factor.

the following conditions: $3 \mathrm{~min}$ of initial denaturation at $95^{\circ} \mathrm{C}$, followed by 40 cycles at $95^{\circ} \mathrm{C}$ for $5 \mathrm{sec}$ (temperature transition rate $4.4^{\circ} \mathrm{C} / \mathrm{sec}$ ) and $60^{\circ} \mathrm{C}$ extension for $25 \mathrm{sec}$. Samples were amplified simultaneously in triplicate in a single assay run. mRNA expression levels are presented as a ratio between the target gene and GAPDH gene expression; the fold-change was calculated using $2^{-\Delta \Delta \mathrm{Cq}}(14)$.

Statistical analysis. Statistical analysis was performed using GraphPad Prism software version 5.0 (GraphPad Software, Inc., La Jolla, CA, USA). All data are expressed as the means \pm standard deviation or standard error of the mean. Data were analyzed using Student's t-test or one-way analysis of variance (ANOVA). Tukey's multiple comparison test was conducted following ANOVA to compare multiple groupse (NSCLC tissues and their adjacent/normaltissues). $\mathrm{P}<0.05$ was considered toindicate a statistically significant difference.

\section{Results}

mRNA expression levels of TF variants in human cell lines and tissues. As shown in Fig. 2A, the mRNA expression levels
flTF were compared with those of asTF in 11 human cell lines, including human cervical cancer (C-33A, HeLa and SiHa), breast cancer (ZR-75-1, MCF-7, BT-474, MDA-MB-468 and MDA-MB-231), hepatoblastoma (HepG2), colorectal cancer (Caco-2) and umbilical vein (EA.hy926) cells. The expression levels were also compared in five types of tissue specimen, including placenta, esophageal cancer, cervical cancer, lung cancer and breast cancer tissues (Fig. 2B). The results demonstrated that fITF and asTF exist in a wide range of human tissues and cells, and the mRNA expression levels of flTF were significantly higher compared with asTF in all samples tested $(\mathrm{P}<0.05)$.

Effects of CDCA on the mRNA expression levels of TF variants in HepG2 and EA.hy926 cells. There was a significant increase in the expression levels of flTF in the HepG2 and EA.hy926 cells treated with CDCA (Fig. 3A). CDCA also promoted the expression of asTF in EA.hy926 cells, but had no significant effect on HepG2 cells (Fig. 3B).

Effects of apoM overexpression on the mRNA expression levels of TF variants in Caco-2 and EA.hy926 cells. Compared with in the NC (empty vector control) group, 
A

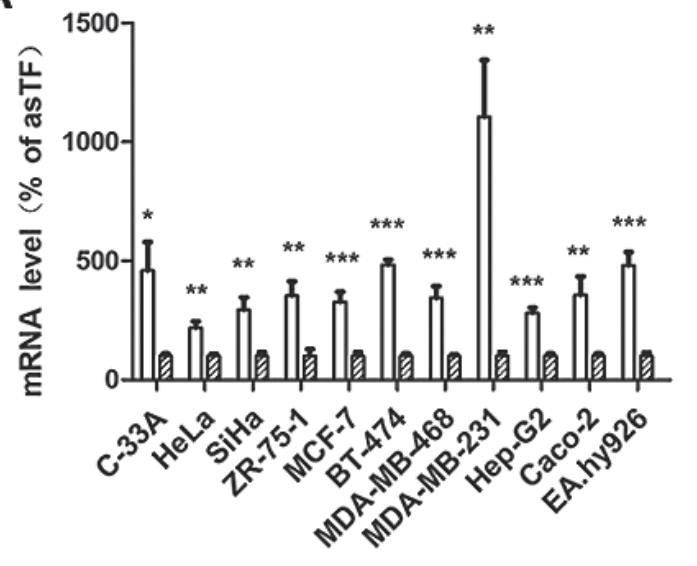

B

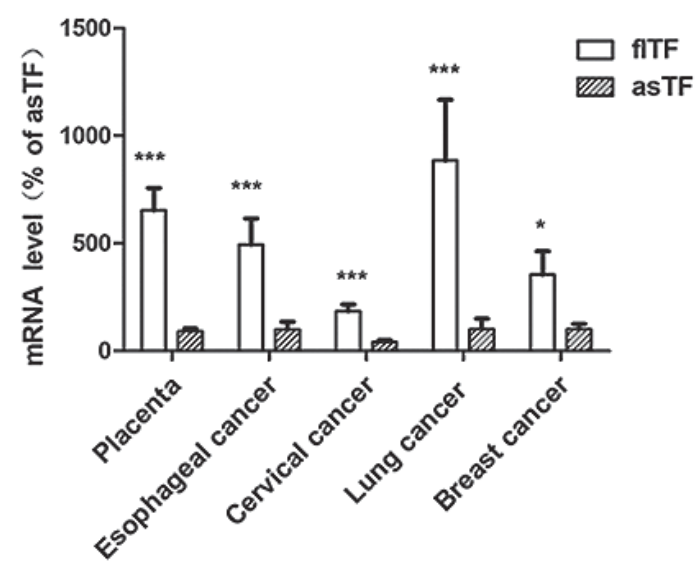

Figure 2. Expression levels of fTTF and asTF in human cell lines and tissues. Quantitative polymerase chain reaction analysis revealed that flTF and asTF mRNA was expressed in (A) all 11 human cell lines tested and (B) all five types of tissue specimens tested. ${ }^{*} \mathrm{P}<0.05,{ }^{* * *} \mathrm{P}<0.01$ and ${ }^{* * * *} \mathrm{P}<0.001 \mathrm{vs}$. asTF. asTF, alternatively spliced TF; fiTF, full-length TF; TF, tissue factor.

A

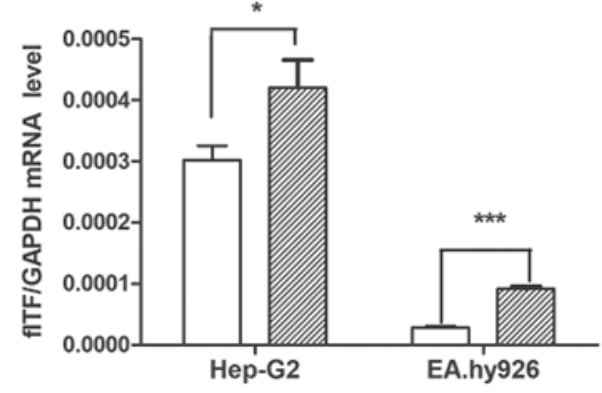

B

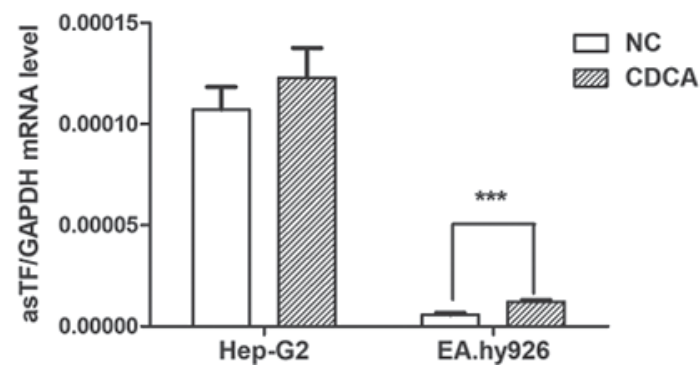

Figure 3. Expression levels of fTTF and asTF in HepG2 and EA.hy 926 cells treated with CDCA (50 $\mu \mathrm{mol})$. mRNA expression levels of (A) fTTF and (B) asTF in the CDCA and NC groups (treated with $0.1 \%$ ethanol) were quantified using quantitative polymerase chain reaction and normalized to those of GAPDH (n=6). ${ }^{*} \mathrm{P}<0.05$ and ${ }^{* * * *} \mathrm{P}<0.001$ vs. NC. asTF, alternatively spliced TF; CDCA, chenodeoxycholic acid; flTF, full-length TF; NC, negative control; TF, tissue factor.

A

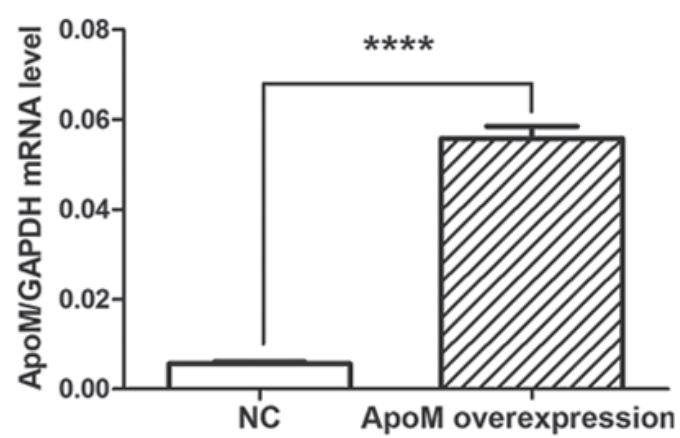

B

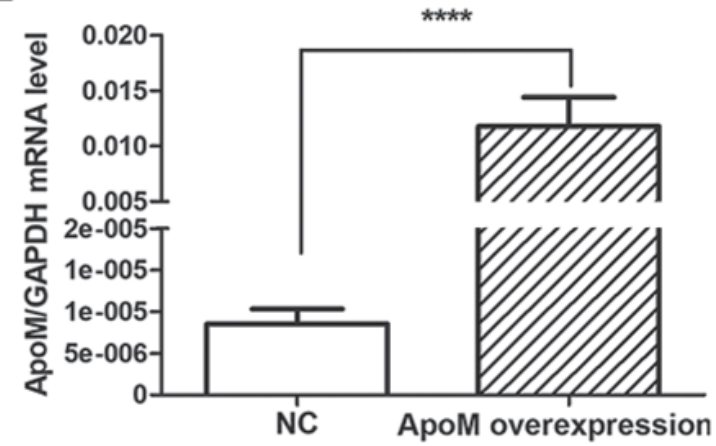

Figure 4. ApoM transduction efficiency. ApoM mRNA expression levels in NC (infected with empty vectors) and overexpression groups (infected with apoM lentivirus vectors) in (A) Caco-2 and (B) EA.hy926 cells, as quantified using quantitative polymerase chain reaction. The mRNA expression levels of apoM were higher in the apoM overexpression group compared with in the $\mathrm{NC}$ group. ${ }^{* * * * *} \mathrm{P}<0.0001$ vs. NC. apoM, apolipoprotein $\mathrm{M}$; NC, negative control.

apoM lentivirus transduction increased apoM expression by 9.95-fold $(\mathrm{P}<0.0001)$ in Caco-2 cells (Fig. 4A) and 1,379.07-fold $(\mathrm{P}<0.0001)$ in EA.hy926 cells (Fig. 4B). As shown in Fig. 5, the results demonstrated that the mRNA expression levels of asTF were increased in Caco-2 cells overexpressing apoM compared with in the NC group $(\mathrm{P}<0.05)$; however, no significant effect was observed on flTF expression in those cells. In addition,
apoM overexpression had no significant effect on fITF and asTF in EA.hy926 cells.

$m R N A$ expression levels of TF variants in tumor, normal and adjacent tissues. To investigate whether the expression of individual splice variants differed during the tumor process, the expression levels of flTF and asTF were investigated in 
Table II. Expression levels of flTF and asTF in cell lines and tissues.

\begin{tabular}{|c|c|c|c|c|c|}
\hline \multirow[b]{2}{*}{ Cell lines and tissues } & \multirow[b]{2}{*}{ Group } & \multicolumn{2}{|l|}{ flTF } & \multicolumn{2}{|l|}{ asTF } \\
\hline & & Expression levels & P-value & Expression levels & P-value \\
\hline \multirow[t]{2}{*}{ Caco-2 } & ApoM OE group & $4.73 \times 10^{-4} \pm 6.22 \times 10^{-5}$ & 0.66 & $2.29 \times 10^{-4} \pm 4.53 \times 10^{-5}$ & $0.03^{\mathrm{a}}$ \\
\hline & Control group & $4.23 \times 10^{-4} \pm 9.22 \times 10^{-5}$ & & $1.19 \times 10^{-4} \pm 1.27 \times 10^{-5}$ & \\
\hline \multirow[t]{2}{*}{ EA.hy926 } & ApoM OE group & $1.40 \times 10^{-4} \pm 1.43 \times 10^{-5}$ & 0.24 & $4.17 \times 10^{-5} \pm 8.43 \times 10^{-6}$ & 0.82 \\
\hline & Control group & $1.59 \times 10^{-4} \pm 3.86 \times 10^{-6}$ & & $4.50 \times 10^{-5} \pm 1.14 \times 10^{-5}$ & \\
\hline \multirow[t]{2}{*}{ HepG2 cells } & CDCA group & $4.20 \times 10^{-4} \pm 4.53 \times 10^{-5}$ & $0.04^{\mathrm{a}}$ & $1.23 \times 10^{-4} \pm 1.48 \times 10^{-5}$ & 0.42 \\
\hline & Control group & $3.02 \times 10^{-4} \pm 2.34 \times 10^{-5}$ & & $1.07 \times 10^{-4} \pm 1.11 \times 10^{-5}$ & \\
\hline \multirow[t]{3}{*}{ NSCLC } & Normal tissues & $0.034 \pm 0.034$ & 0.97 & $0.005 \pm 0.008$ & 0.54 \\
\hline & Adjacent tissues & $0.052 \pm 0.067$ & & $0.011 \pm 0.016$ & \\
\hline & Tumor tissues & $0.040 \pm 0.041$ & & $0.008 \pm 0.015$ & \\
\hline \multirow[t]{2}{*}{ Cervical cancer } & Normal tissues & $0.007 \pm 0.006$ & 0.62 & $0.0003 \pm 0.0004$ & $0.001^{\mathrm{a}}$ \\
\hline & Tumor tissues & $0.007 \pm 0.007$ & & $0.002 \pm 0.002$ & \\
\hline
\end{tabular}

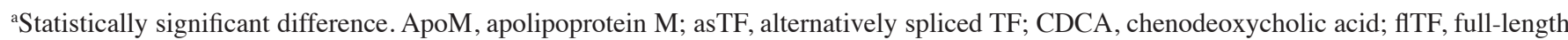
TF; NSCL, non-small cell lung cancer; OE, overexpression; TF, tissue factor.

A

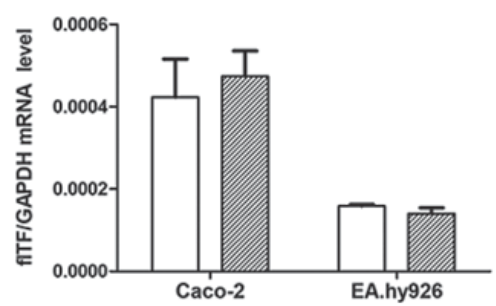

B

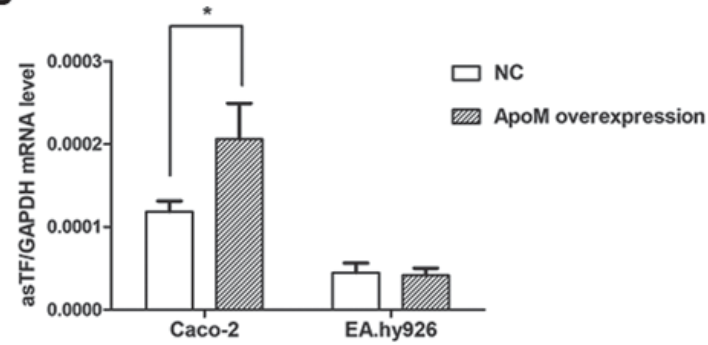

Figure 5. Effects of apoM overexpression on the mRNA expression levels of fiTF and asTF in Caco-2 and EA.hy926 cells. (A) flTF and (B) asTF mRNA expression levels in the apoM overexpression and $\mathrm{NC}$ groups were quantified using quantitative polymerase chain reaction. No significant effect on flTF was observed in cells overexpressing apoM. In addition, apoM overexpression promoted the mRNA expression levels of asTF in Caco-2 cells, but had was no significant effect on asTF expression in EA.hy926 cells. * $\mathrm{P}<0.05$ vs. NC. apoM, apolipoprotein M; asTF, alternatively spliced TF; flTF, full length TF; NC, negative control; TF, tissue factor.

A

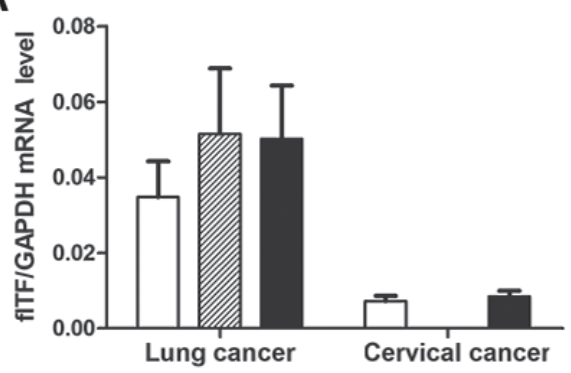

B

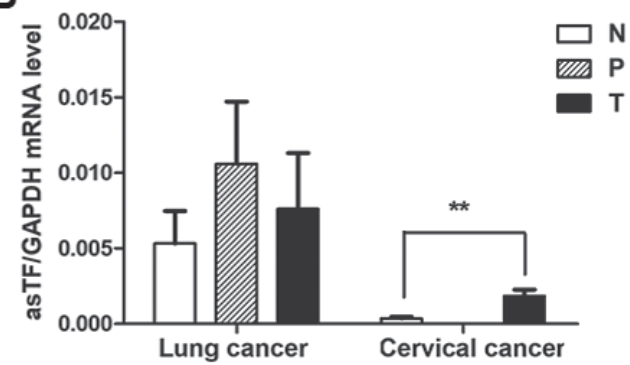

Figure 6. mRNA expression levels of flTF and asTF in NSCLC and cervical cancer tissues. NSCLC tissues were paired, whereas cervical tissues were unpaired due to the lack of paracancerous tissues. (A) flTF and (B) asTF mRNA expression levels in the lung and cervical cancer tissues and adjacent/normal tissues were quantified using quantitative polymerase chain reaction. No significant effect was observed on the mRNA expression levels of flTF among the lung and cervical tissues and their adjacent/normal tissues. The expression levels of asTF were higher in cervical cancer tissues compared with in the normal group, whereas there was no significant difference in asTF expression between lung cancer and adjacent/normal tissues. ${ }^{* *} \mathrm{P}<0.01$. asTF, alternatively spliced TF; flTF, full length TF; N, normal tissue; NSCLC, non-small cell lung cancer; P, paracancerous tissue; T, tumor tissue; TF, tissue factor.

cervical and lung cancer specimens. As shown in Fig. 6, there was no significant difference in the mRNA expression levels of flTF and asTF splice variants among any of the lung cancer tissues. There was also no significant difference in the mRNA expression levels of flTF between cervical cancer and normal cervical tissues; however, asTF was significantly increased 


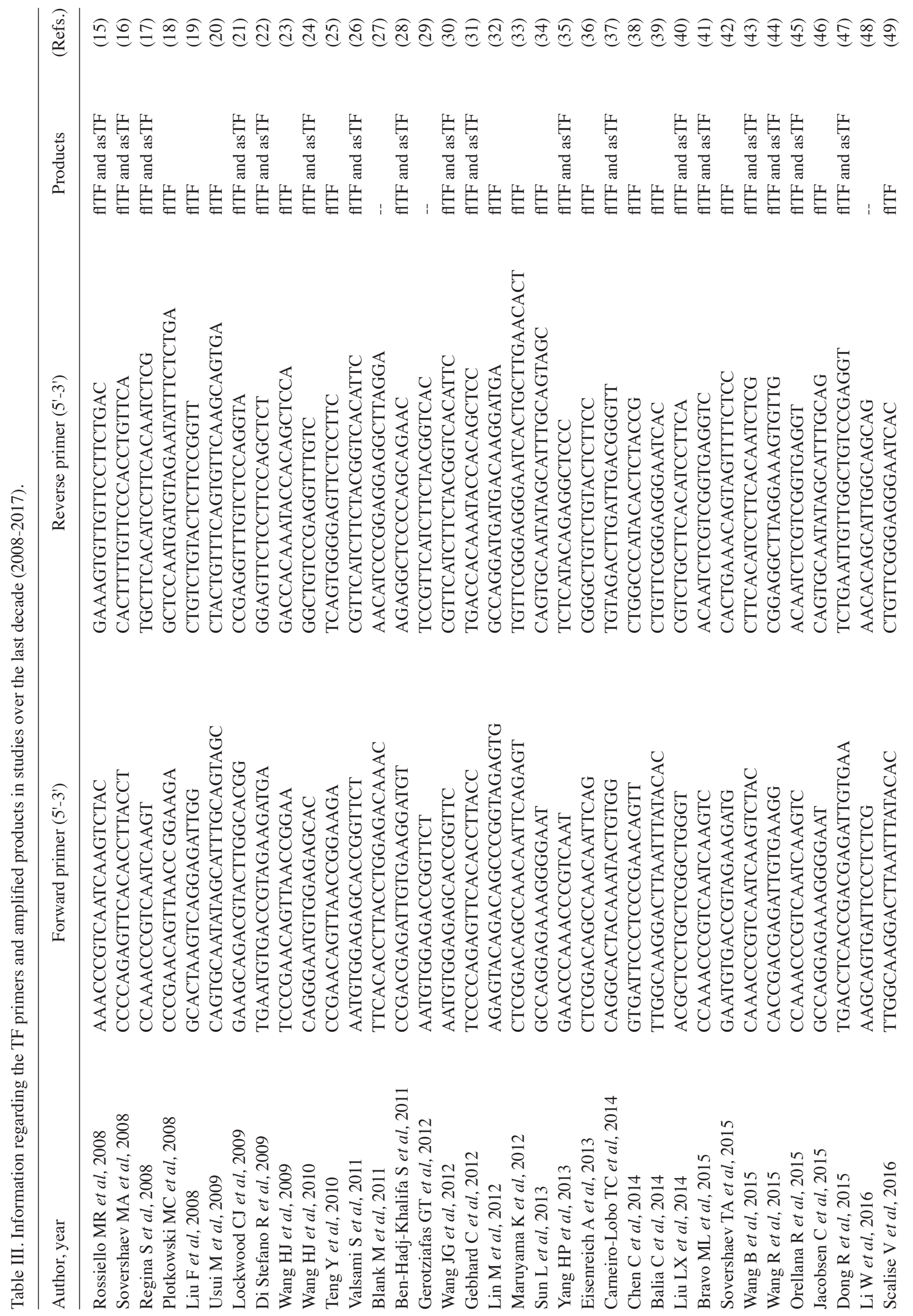


in cervical cancer tissues compared with in normal cervical tissues $(\mathrm{P}<0.01)$.

Summary of the negative results. Overexpression of apoM had little effect on the mRNA expression levels of flTF in the Caco-2 and EA.hy926 cells. In addition, there was no significant difference between the mRNA expression levels of flTF in the adjacent/normal and tumor NSCLC and cervical cancer tissues (Table II). With regards to asTF mRNA expression, CDCA had little effect on HepG2 cells and apoM overexpression did not affect the levels of asTF in the EA.hy926 cells. Similarly, there was no statistically significant difference in asTF mRNA levels between the adjacent and tumor NSCLC tissues.

\section{Discussion}

Since asTF and flTF serve important and often distinct roles in various biological processes, it is appropriate to study them separately. However, as shown in Table III (15-52), total TF, including asTF and fITF, or flTF alone, has been investigated in studies over the last decade. Few studies have been conducted that have detected asTF and fITF levels separately, according to the primer sequences that have been designed. Furthermore, the primers used in three of these previous studies $(27,29,48)$ are not completely matched with the TF sequence and therefore cannot be used to amplify asTF or flTF.

In terms of expression, as the major form of TF, the mRNA expression levels of flTF were higher than those of asTF in all specimens tested in the present study. Furthermore, in terms of function, CDCA increased the expression levels of flTF in HepG2 cells, whereas those of asTF were not affected. Therefore, it was hypothesized that fITF may contribute to the onset of liver cancer. The present study also demonstrated that CDCA increased the expression levels of fITF and asTF in EA.hy926 cells, which indicated that CDCA may be associated with vasoconstriction. Furthermore, Kundu et al (9) demonstrated that CDCA is capable of promoting vessel formation. In a previous study, both variants were revealed to mediate various physiological and pathological functions, including angiogenesis (2). Therefore, CDCA may promote vessel formation through the upregulation of these two variants; however, the possible mechanism requires further investigation.

asTF is associated with the development of numerous types of cancer. A previous study demonstrated that the mRNA expression levels of apoM in colorectal cancer tissues were significantly increased in patients with lymph node metastasis (53). The present study revealed that the expression levels of asTF were increased in Caco- 2 cells that overexpressed apoM compared with the control cells. Conversely, overexpression of apoM had little effect on flTF. Consistent with this result, Yu et al (54) demonstrated that flTF expression in colorectal cancer has no influence on cell proliferation in vitro; however, asTF has been reported to promote cell proliferation in vitro and tumor growth in vivo $(1,36,55)$. It may therefore be hypothesized that asTF, not flTF, contributes to the onset of cancer.

Venteclef et al (56) demonstrated that bile acids suppress apoM expression in vitro and in vivo. The results of the present study demonstrated that as one of the bile acids, CDCA increased the expression levels of the two TF variants in 
the EA.hy926 cells and apoM increased as TF expression in Caco- 2 cells. These data suggested that apoM may be involved in the regulation of TF expression induced by CDCA, but this requires further investigation.

The mRNA expression levels of flTF were not significantly different between the cervical cancer and normal tissues; however, those of asTF were markedly increased in cancer tissues. Previous studies regarding the coagulant properties of asTF have been fairly inconclusive $(57,58)$, but they are likely to be essential during angiogenesis associated with the development of cancer $(59,60)$. The present study demonstrated that the measurement of asTF mRNA may be associated with cervical cancer risk. The expression levels of flTF and asTF in lung cancer and paracancerous tissues were higher compared with in normal control tissues; however, the differences were not statistically significant. Rollin et al (61) demonstrated that the relative amount of asTF is low. This previous study also analyzed the levels of asTF in NSCLC tumors according to clinicopathological features; the results revealed that there is no association between asTF and sex, age, stage and differentiation grade, yet patients with high asTF tumor mRNA expression had a poorer prognosis. Therefore, further studies are required to investigate the correlation between TF and NSCLC tumors.

A limitation of the present study is that control or paired normal esophageal and breast cancer tissues samples were not analyzed. Therefore, the role of flTF and asTF in these two types of cancer could not be clearly identified.

In conclusion, TF isoforms are able to activate distinct signaling pathways (2), leading to the modulation of cancer-associated biological processes and nonhemostatic pathophysiological processes, including thrombosis, angiogenesis, tumor growth and metastasis. A previous study demonstrated that fITF mediates cell signaling via protease activated receptor 2 and downstream signaling proteins, including protein kinase $\mathrm{C}$ and extracellular signal-regulated kinase 1 and 2, whereas asTF exhibits activity via integrin ligation $(2,62)$. To the best of our knowledge, the present study is the first to reveal that flTF expression may be increased compared with asTF in all tissue specimens tested, and suggested that overexpression of apoM and CDCA may affect the mRNA expression levels of the two variants. Furthermore, the expression levels of the two variants may be different in the same cancer tissues. These results provide further information regarding the TF system and emphasize the significance of flTF and asTF expression in tumor progression and other types of disease.

\section{Acknowledgements}

Not applicable.

\section{Funding}

The present study was supported by the Changzhou High-Level Medical Talents Training Project (grant no. 2016ZCLJ002).

\section{Availability of data and materials}

All data generated or analyzed during the present study are included in this published article.

\section{Authors' contributions}

GL and NX contributed to the design of the study and revision of the manuscript. LP performed the experiments, analyzed the data and contributed to writing the manuscript. YY and QM performed experiments. MY and SY were responsible for experiments and statistical analysis.

\section{Ethics approval and consent to participate}

The experimental protocols were approved by the Institutional Ethics Committee of the Third Affiliated Hospital of Soochow University and all patients provided written informed consent for this study.

\section{Patient consent for publication}

Written informed consent was obtained from all patients.

\section{Competing interests}

All authors declare that they have no competing interests.

\section{References}

1. Eisenreich A, Boltzen U, Malz R, Schultheiss HP and Rauch U: Overexpression of alternatively spliced tissue factor induces the pro-angiogenic properties of murine cardiomyocytic HL-1 cells. Circ J 75: 1235-1242, 2011.

2. Leppert $U$ and Eisenreich A: The role of tissue factor isoforms in cancer biology. Int J Cancer 137: 497-503, 2015.

3. Boltzen U, Eisenreich A, Antoniak S, Weithaeuser A Fechner H, Poller W, Schultheiss HP, Mackman N and Rauch U: Alternatively spliced tissue factor and full-length tissue factor protect cardiomyocytes against TNF- $\alpha$-induced apoptosis. J Mol Cell Cardiol 52: 1056-1065, 2012.

4. Eisenreich A: Regulation of vascular function on posttranscriptional level. Thrombosis 2013: 948765, 2013.

5. van den Berg YW, van den Hengel LG, Myers HR, Ayachi O, Jordanova E, Ruf W, Spek CA, Reitsma PH, Bogdanov VY and Versteeg HH: Alternatively spliced tissue factor induces angiogenesis through integrin ligation. Proc Natl Acad Sci USA 106: 19497-19502, 2009.

6. Chand HS, Ness SA and Kisiel W: Identification of a novel human tissue factor splice variant that is upregulated in tumor cells. Int J Cancer 118: 1713-1720, 2006.

7. Eisenreich A, Bogdanov VY, Zakrzewicz A, Pries A, Antoniak S, Poller W, Schultheiss HP and Rauch U: Cdc2-like kinases and DNA topoisomerase I regulate alternative splicing of tissue factor in human endothelial cells. Circ Res 104: 589-599, 2009.

8. Han X, Guo B, Li Y and Zhu B: Tissue factor in tumor microenvironment: A systematic review. J Hematol Oncol 7: 54, 2014.

9. Kundu S, Bansal S, Muthukumarasamy KM, Sachidanandan C, Motiani RK and Bajaj A: Deciphering the role of hydrophobic and hydrophilic bile acids in angiogenesis using in vitro and in vivo model systems. Medchemcomm 8: 2248-2257, 2017.

10. Xu N and Dahlbäck B: A novel human apolipoprotein (apoM). J Biol Chem 274: 31286-31290, 1999.

11. Ahmad A, Sundquist K, Zöller B, Dahlbäck B, Svensson PJ, Sundquist J and Memon AA: Identification of polymorphisms in apolipoprotein $\mathrm{M}$ gene and their relationship with risk of recurrent venous thromboembolism. Thromb Haemost 116: 432-441, 2016.

12. Nádró B, Juhász L, Szentpéteri A, Páll D, Paragh G and Harangi $\mathrm{M}$ : The role of apolipoprotein $\mathrm{M}$ and sphingosine 1-phosphate axis in the prevention of atherosclerosis. Orv Hetil 159: 168-175, 2018 (In Hungarian).

13. Zhu Y, Luo G, Jiang B, Yu M, Feng Y, Wang M, Xu N and Zhang X: Apolipoprotein $\mathrm{M}$ promotes proliferation and invasion in non-small cell lung cancers via upregulating S1PR1 and activating the ERK1/2 and PI3K/AKT signaling pathways. Biochem Biophys Res Commun 501: 520-526, 2018. 
14. Livak KJ and Schmittgen TD: Analysis of relative gene expression data using real-time quantitative PCR and the 2(-Delta Delta C(T)) method. Methods 25: 402-408, 2001.

15. Rossiello MR, Rotunno C, Coluccia A, Carratù MR, Di Santo A Evangelista V, Semeraro N and Colucci M: Ochratoxin A inhibits the production of tissue factor and plasminogen activator inhibitor-2 by human blood mononuclear cells: Another potential mechanism of immune-suppression. Toxicol Appl Pharmacol 229: 227-231, 2008.

16. Sovershaev MA, Lind KF, Devold H, Jørgensen TØ, Hansen JB, Østerud B and Egorina EM: No evidence for the presence of tissue factor in high-purity preparations of immunologically isolated eosinophils. J Thromb Haemost 6: 1742-1749, 2008.

17. Regina S, Rollin J, Bléchet C, Iochmann S, Reverdiau P and Gruel Y: Tissue factor expression in non-small cell lung cancer: Relationship with vascular endothelial growth factor expression, microvascular density, and K-ras mutation. J Thorac Oncol 3 : 689-697, 2008

18. Plotkowski MC, Feliciano LF, Machado GB, Cunha LG Jr, Freitas C, Saliba AM and de Assis MC: ExoU-induced procoagulant activity in pseudomonas aeruginosa-infected airway cells. Eur Respir J 32: 1591-1598, 2008.

19. Liu F, Huang R, Yao J, Wei W, Hu Y, Song S and Li J: Homocysteine-induced enhanced expression of tissue factor in human vascular smooth muscle cells. J Huazhong Univ Sci Technolog Med Sci 28: 520-524, 2008.

20. Usui M, Kuriyama N, Kisawada M, Hamada T, Mizuno S, Sakurai H, Tabata M, Imai $H$, Okamoto $K$, Uemoto $S$ and Isaji S: Tissue factor expression demonstrates severe sinusoidal endothelial cell damage during rejection after living-donor liver transplantation. J Hepatobiliary Pancreat Surg 16: 513-520, 2009

21. Lockwood CJ, Murk W, Kayisli UA, Buchwalder LF, Huang ST, Funai EF, Krikun G and Schatz F: Progestin and thrombin regulate tissue factor expression in human term decidual cells. J Clin Endocrinol Metab 94: 2164-2170, 2009

22. Di Stefano R, Barsotti MC, Armani C, Santoni T, Lorenzet R, Balbarini A and Celi A: Human peripheral blood endothelial progenitor cells synthesize and express functionally active tissue factor. Thromb Res 123: 925-930, 2009.

23. Wang HJ, Huang H, Chuang YC and Huang HC: Paclitaxel induces up-regulation of tissue factor in human aortic endothelial cells. Int Immunopharmacol 9: 144-147, 2009.

24. Wang HJ, Lo WY, Lu TL and Huang H: (-)-Epigallocatechin3-gallate decreases thrombin/paclitaxel-induced endothelial tissue factor expression via the inhibition of c-Jun terminal NH2 kinase phosphorylation. Biochem Biophys Res Commun 391 716-721, 2010.

25. Teng Y, Jiang R, Lin Q, Ding C and Ye Z: The relationship between plasma and placental tissue factor, and tissue factor pathway inhibitors in severe pre-eclampsia patients. Thromb Res 126: e41-45, 2010.

26. Valsami S, Ruf W, Leikauf MS, Madon J, Kaech A and Asmis LM: Immunomodulatory drugs increase endothelial tissue factor expression in vitro. Thromb Res 127: 264-271, 2011

27. Blank M,BaraamL,Eisenstein M,Fridkin M,DardikR,Heldman Y, Katchalski-Katzir E and Shoenfeld Y: $\beta 2-G l y c o p r o t e i n-I$ based peptide regulate endothelial-cells tissue-factor expression via negative regulation of pGSK $3 \beta$ expression and reduces experimental-antiphospholipid-syndrome. J Autoimmun 37: 8-17, 2011.

28. Ben-Hadj-Khalifa S, Hézard N, Almawi WY, Lakbakbi S, Macé C, Cornillet-Lefebvre P, Mahjoub T and Nguyen P: IL-10 modulates fondaparinux inhibition of monocyte-induced thrombin generation. J Thromb Thrombolysis 32: 311-317, 2011

29. Gerotziafas GT, Galea V, Mbemba E, Khaterchi A, Sassi M, Baccouche H, Prengel C, van Dreden P, Hatmi M, Bernaudin JF and Elalamy I: Tissue factor over-expression by human pancreatic cancer cells BXPC3 is related to higher prothrombotic potential as compared to breast cancer cells MCF7. Thromb Res 129: 779-786, 2012

30. Wang JG, Geddings JE, Aleman MM, Cardenas JC, Chantrathammachart P, Williams JC, Kirchhofer D, Bogdanov VY, Bach RR, Rak J, et al: Tumor-derived tissue factor activates coagulation and enhances thrombosis in a mouse xenograft model of human pancreatic cancer. Blood 119: 5543-5552, 2012.

31. Gebhard C, Holy EW, Camici GG, Akhmedov A, Stämpfli SF, Stähli BE, von Rickenbach B, Breitenstein A, Greutert H, Yang Z, et al: Caffeine induces endothelial tissue factor expression via phosphatidylinositol 3-kinase inhibition. Thromb Haemost 107: 884-894, 2012.
32. Lin M, Weng H, Wang X, Zhou B, Yu P and Wang Y: The role of tissue factor and protease-activated receptor 2 in endometriosis. Am J Reprod Immunol 68: 251-257, 2012.

33. Maruyama K, Morishita E, Yuno T, Sekiya A, Asakura H, Ohtake S and Yachie A: Carbon monoxide (CO)-releasing molecule-derived $\mathrm{CO}$ regulates tissue factor and plasminogen activator inhibitor type 1 in human endothelial cells. Thromb Res 130: e188-193, 2012.

34. Sun L, Liu Y, Lin S, Shang J, Liu J, Li J, Yuan S and Zhang L: Early growth response gene-1 and hypoxia-inducible factor-1 $\alpha$ affect tumor metastasis via regulation of tissue factor. Acta Oncol 52: 842-851, 2013.

35. Yang HP, Yue L, Jiang WW, Liu Q, Kou JP and Yu BY: Diosgenin inhibits tumor necrosis factor-induced tissue factor activity and expression in THP-1 cells via down-regulation of the NF- $\mathrm{B}$, Akt, and MAPK signaling pathways. Chin J Nat Med 11: 608-615, 2013

36. Eisenreich A, Zakrzewicz A, Huber K, Thierbach H, Pepke W, Goldin-Lang P, Schultheiss HP, Pries A and Rauch U: Regulation of pro-angiogenic tissue factor expression in hypoxia-induced human lung cancer cells. Oncol Rep 30: 462-470, 2013.

37. Carneiro-Lobo TC, Lima MT, Mariano-Oliveira A, Dutra-Oliveira A, Oba-Shinjo SM, Marie SK, Sogayar MC and Monteiro RQ: Expression of tissue factor signaling pathway elements correlates with the production of vascular endothelial growth factor and interleukin-8 in human astrocytoma patients. Oncol Rep 31: 679-686, 2014.

38. Chen C, Liao D, Wang J, Liang Z and Yao Q: Anti-human protein $\mathrm{S}$ antibody induces tissue factor expression through a direct interaction with platelet phosphofructokinase. Thromb Res 133: 222-228, 2014.

39. Balia C, Petrini S, Scalise V, Neri T, Carnicelli V, Cianchetti S, Zucchi R, Celi A and Pedrinelli R: Compound 21, a selective angiotensin II type 2 receptor agonist, downregulates lipopolysaccharide-stimulated tissue factor expression in human peripheral blood mononuclear cells. Blood Coagul Fibrinolysis 25: 501-506, 2014.

40. Liu LX, Zeng H, Liu EY and Chen FP: Tissue factor expression and methylation regulation in differentiation of embryonic stem cells into trophoblast. Asian Pac J Trop Med 7: 557-561, 2014.

41. Bravo ML, Pinto MP, Gonzalez I, Oliva B, Kato S, Cuello MA, Lange CA and Owen GI: Progesterone regulation of tissue factor depends on MEK1/2 activation and requires the proline-rich site on progesterone receptor. Endocrine 48: 309-320, 2015.

42. Sovershaev TA, Egorina EM, Unruh D, Bogdanov VY, Hansen JB and Sovershaev MA: BMP-7 induces TF expression in human monocytes by increasing F3 transcriptional activity. Thromb Res 135: 398-403, 2015.

43. Wang B, Xiong S, Hua Q, Chen C, Liao H, Chen L, Yao W, Wu D and Tao Z: Tissue factor is strongly expressed in pericarcinomatous tissue in patients with laryngeal carcinoma. Int J Clin Exp Pathol 8: 13719-13724, 2015.

44. Wang R, Ma YT, Ma J, Chen D, Wu B, Wen BZ and He SL: Simultaneous detection of peripheral mononuclear cell and plasma tissue factor expression for prevention and treatment of ischemic cardiocerebrovascular diseases. Int J Clin Exp Pathol 8: 5674-5680, 2015

45. Orellana R, Kato S, Erices R, Bravo ML, Gonzalez P, Oliva B, Cubillos S, Valdivia A, Ibañez C, Brañes J, et al: Platelets enhance tissue factor protein and metastasis initiating cell markers, and act as chemoattractants increasing the migration of ovarian cancer cells. BMC Cancer 15: 290, 2015.

46. Jacobsen C, Oechsle K, Hauschild J, Steinemann G, Spath B, Bokemeyer C, Ruf W, Honecker F and Langer F: Regulation of tissue factor in NT2 germ cell tumor cells by cisplatin chemotherapy. Thromb Res 136: 673-681, 2015.

47. Dong R, Chen W, Feng W, Xia C, Hu D, Zhang Y, Yang Y, Wang DW, Xu X and Tu L: Exogenous bradykinin inhibits tissue factor induction and deep vein thrombosis via activating the eNOS/phosphoinositide 3-kinase/Akt signaling pathway. Cell Physiol Biochem 37: 1592-1606, 2015.

48. Li W, Chen W, Xie M, Huang H, Su H, Han H, Zhang D, Zhang Y, Yang X, Xu W, et al: Fasudil inhibits tissue factor and plasminogen activator inhibitor-1 secretion by peripheral blood mononuclear cells in CAPD patients. Ren Fail 38: 1359-1363, 2016.

49. Scalise V, Balia C, Cianchetti S, Neri T, Carnicelli V, Zucchi R, Franzini M, Corti A, Paolicchi A, Celi A and Pedrinelli R: Non enzymatic upregulation of tissue factor expression by gamma-glutamyl transferase in human peripheral blood mononuclear cells. Thromb J 14: 45, 2016. 
50. Krychtiuk KA, Kaun C, Hohensinner PJ, Stojkovic S, Seigner J, Kastl SP, Zuckermann A, Eppel W, Rauscher S, de Martin R, et al: Anti-thrombotic and pro-fibrinolytic effects of levosimendan in human endothelial cells in vitro. Vascul Pharmacol 90: 44-50, 2017.

51. Gao H, Liu L, Zhao Y, Hara H, Chen P, Xu J, Tang J, Wei L, Li Z, Cooper DKC, et al: Human IL-6, IL-17, IL-1 $\beta$, and TNF- $\alpha$ differently regulate the expression of pro-inflammatory related genes, tissue factor, and swine leukocyte antigen class I in porcine aortic endothelial cells. Xenotransplantation: doi.org/10.1111/xen.12291.

52. Brambilla M, Rossetti L, Zara C, Canzano P, Giesen PLA Tremoli E and Camera M: Do methodological differences account for the current controversy on tissue factor expression in platelets? Platelets 29: 406-414, 2018

53. Luo G, Zhang $\mathrm{X}$, Mu Q, Chen L, Zheng L, Wei J, Berggren-Söderlund M, Nilsson-Ehle P and Xu N: Expression and localization of apolipoprotein $\mathrm{M}$ in human colorectal tissues. Lipids Health Dis 9: 102, 2010.

54. Yu JL, May L, Lhotak V, Shahrzad S, Shirasawa S, Weitz JI, Coomber BL, Mackman N and Rak JW: Oncogenic events regulate tissue factor expression in colorectal cancer cells: Implications for tumor progression and angiogenesis. Blood 105: 1734-1741, 2005.

55. Kocatürk B, Van den Berg YW, Tieken C, Mieog JS, de Kruijf EM, Engels CC, van der Ent MA, Kuppen PJ, Van de Velde CJ, Ruf W, et al: Alternatively spliced tissue factor promotes breast cancer growth in a $\beta 1$ integrin-dependent manner. Proc Natl Acad Sci USA 110: 11517-11522, 2013.

56. Venteclef N, Haroniti A, Tousaint JJ, Talianidis I and Delerive P: Regulation of anti-atherogenic apolipoprotein $\mathrm{M}$ gene expression by the orphan nuclear receptor LRH-1. J Biol Chem 283: 3694-3701, 2008.
57. Censarek P, Bobbe A, Grandoch M, Schrör K and Weber AA: Alternatively spliced human tissue factor (asHTF) is not pro-coagulant. Thromb Haemost 97: 11-14, 2007.

58. Szotowski B, Antoniak S, Poller W, Schultheiss HP and Rauch U: Procoagulant soluble tissue factor is released from endothelial cells in response to inflammatory cytokines. Circ Res 96: 1233-1239, 2005.

59. Mackman N and Davis GE: Blood coagulation and blood vessel development: Is tissue factor the missing link? Arterioscler Thromb Vasc Biol 31: 2364-2366, 2011.

60. Unruh D, Turner K, Srinivasan R, Kocatürk B, Qi X, Chu Z, Aronow BJ, Plas DR, Gallo CA, Kalthoff H, et al: Alternatively spliced tissue factor contributes to tumor spread and activation of coagulation in pancreatic ductal adenocarcinoma. Int J Cancer 134: 9-20, 2014.

61. Rollin J, Regina S and Gruel Y: Tumor expression of alternatively spliced tissue factor is a prognostic marker in non-small cell lung cancer. J Thromb Haemost 8: 607-610, 2010.

62. Giannarelli C, Alique M, Rodriguez DT, Yang DK, Jeong D, Calcagno C, Hutter R, Millon A, Kovacic JC, Weber T, et al: Alternatively spliced tissue factor promotes plaque angiogenesis through the activation of hypoxia-inducible factor- $1 \alpha$ and vascular endothelial growth factor signaling. Circulation 130: 1274-1286, 2014

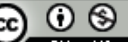

This work is licensed under a Creative Commons Attribution 4.0 International (CC BY-NC 4.0) License 